\title{
Precision measurement of $D$ meson mass differences
}

\section{LHCD}

\section{The LHCb collaboration}

E-mail: Matthew.Needham@cern.ch

ABSTRACT: Using three- and four-body decays of $D$ mesons produced in semileptonic $b$ hadron decays, precision measurements of $D$ meson mass differences are made together with a measurement of the $D^{0}$ mass. The measurements are based on a dataset corresponding to an integrated luminosity of $1.0 \mathrm{fb}^{-1}$ collected in $p p$ collisions at $7 \mathrm{TeV}$. Using the decay $D^{0} \rightarrow K^{+} K^{-} K^{-} \pi^{+}$, the $D^{0}$ mass is measured to be

$$
M\left(D^{0}\right)=1864.75 \pm 0.15 \text { (stat) } \pm 0.11 \text { (syst) } \mathrm{MeV} / c^{2} .
$$

The mass differences

$$
\begin{aligned}
& M\left(D^{+}\right)-M\left(D^{0}\right)=4.76 \pm 0.12(\text { stat }) \pm 0.07 \text { (syst) } \mathrm{MeV} / c^{2}, \\
& M\left(D_{s}^{+}\right)-M\left(D^{+}\right)=98.68 \pm 0.03(\text { stat }) \pm 0.04(\text { syst }) \mathrm{MeV} / c^{2}
\end{aligned}
$$

are measured using the $D^{0} \rightarrow K^{+} K^{-} \pi^{+} \pi^{-}$and $D_{(s)}^{+} \rightarrow K^{+} K^{-} \pi^{+}$modes.

KeYwORDS: Hadron-Hadron Scattering

ARXIV EPRINT: 1304.6865 


\section{Contents}

1 Introduction 1

2 Detector and dataset $\quad 2$

3 Selection 3

4 Fit results 4

$\begin{array}{lll}5 & \text { Systematic uncertainties } & 7\end{array}$

$\begin{array}{llr}6 & \text { Summary } & 8\end{array}$

$\begin{array}{ll}\text { The LHCb collaboration } & 13\end{array}$

\section{Introduction}

Mesons are colourless objects composed of a quark-antiquark pair bound via the strong interaction. Measurements of meson masses provide observables that can be compared to theoretical predictions. For the case of $B$ mesons, precision measurements have been reported in recent years by several experiments [1-3]. In contrast, few precision $D$ meson mass measurements exist.

For the $D^{0}$ meson $^{1}$ the current average of $M\left(D^{0}\right)=1864.91 \pm 0.17 \mathrm{MeV} / c^{2}$, quoted by the Review of Particle Physics [4], is dominated by the measurements of the CLEO [5] and KEDR [6] collaborations. Current knowledge of the masses of the $D^{+}$and $D_{s}^{+}$ mesons, and the mass splitting between these states, is more limited. The most precise determination of the $D^{+}$mass is made by the KEDR collaboration [6] resulting in $M\left(D^{+}\right)=$ $1869.53 \pm 0.49$ (stat) \pm 0.20 (syst) $\mathrm{MeV} / c^{2}$. In addition, two measurements of the mass splitting between the $D^{+}$and $D^{0}$ mesons by the MRK2 [7] and LGW [8] collaborations have been reported. These are averaged [4] to give $M\left(D^{+}\right)-M\left(D^{0}\right)=4.76 \pm 0.28 \mathrm{MeV} / c^{2}$. No absolute measurement of the $D_{s}^{+}$mass with a precision better than the $\mathrm{MeV} / c^{2}$ level exists and the reported values are not in good agreement [4]. More precise measurements of the mass difference relative to the $D^{+}$meson have been reported by several collaborations [913]. These are averaged [4] to give $M\left(D_{s}^{+}\right)-M\left(D^{+}\right)=98.85 \pm 0.25 \mathrm{MeV} / c^{2}$. The fit of open charm mass data [4] leads to $M\left(D_{s}^{+}\right)=1968.49 \pm 0.32 \mathrm{MeV} / c^{2}$. Though this value is significantly more precise than the direct measurement, it would still dominate the systematic uncertainty on the measurement of the $B_{c}^{+}$mass in the $B_{c}^{+} \rightarrow J / \psi D_{s}^{+}$decay mode [14].

\footnotetext{
${ }^{1}$ The inclusion of charge conjugate states is implied.
} 
Recent interest in the $D^{0}$ mass has been driven by the observation of the $X(3872)$ state, first measured by the Belle experiment [15] and subsequently confirmed elsewhere [16-20]. This state, with $J^{P C}=1^{++}[21]$, does not fit well into the quark model picture, and exotic interpretations have been suggested: for example that it is a tetraquark [22] or a loosely bound deuteron-like $D^{* 0} \bar{D}^{0}$ 'molecule' [23]. For the latter interpretation to be valid, the mass of the $X(3872)$ state should be less than the sum of the $D^{* 0}$ and $D^{0}$ masses. Using the fitted value of the $D^{0}$ mass and the measured values for the other quantities quoted in ref. [4], the binding energy $\left(E_{B}\right)$ in this interpretation can be estimated to be

$$
\begin{aligned}
E_{\mathrm{B}} & =M\left(D^{0} D^{* 0}\right)-M(X(3872)) \\
& =2 M\left(D^{0}\right)+\Delta M\left(D^{* 0}-D^{0}\right)-M(X(3872)) \\
& =0.16 \pm 0.32 \mathrm{MeV} / c^{2} .
\end{aligned}
$$

Therefore, the issue of whether the $X(3872)$ can be a bound molecular state remains open. To clarify the situation, more precise measurements of both the $X(3872)$ and $D^{0}$ masses are needed.

In this paper, a measurement of the $D^{0}$ mass using the $D^{0} \rightarrow K^{+} K^{-} K^{-} \pi^{+}$decay mode is reported. This mode has a relatively low energy release, $Q$-value, defined as the difference between the mass of the $D$ meson and the sum of the masses of the daughter particles. Consequently, systematic uncertainties due to the calibration of the momentum scale of the detector are reduced. Other four-body $D^{0}$ decay modes are used to provide a cross-check of the result. In addition, precision measurements of the $D^{+}-D^{0}$ and $D_{s}^{+}-D^{+}$ mass differences are made. For the mass difference measurements the $D^{0} \rightarrow K^{+} K^{-} \pi^{+} \pi^{-}$ mode is used, together with the $D_{(s)}^{+} \rightarrow K^{+} K^{-} \pi^{+}$decay, since these modes have similar $Q$-values.

\section{Detector and dataset}

The analysis uses data, corresponding to an integrated luminosity of $1.0 \mathrm{fb}^{-1}$, collected in $p p$ collisions at a centre-of-mass energy of $\sqrt{s}=7 \mathrm{TeV}$ by the LHCb experiment during 2011. The detector response is studied using a simulation. Proton-proton collisions are generated using Pyтнia 6.4 [24] with the configuration described in ref. [25]. Particle decays are then simulated by EVTGEN [26] in which final state radiation is generated using Рнотоs [27]. The interaction of the generated particles with the detector and its response are implemented using the GEANT4 toolkit [28, 29] with the settings described in ref. [30].

The LHCb detector [31] is a single-arm forward spectrometer covering the pseudorapidity range $2<\eta<5$. It includes a high precision tracking system consisting of a silicon-strip vertex detector surrounding the $p p$ interaction region, a large-area silicon-strip detector located upstream of a dipole magnet with a bending power of about $4 \mathrm{Tm}$, and three stations of silicon-strip detectors and straw drift tubes placed downstream. The polarity of the dipole magnet is reversed at intervals that correspond to roughly $0.1 \mathrm{fb}^{-1}$ of collected data in order to minimize systematic uncertainties. The combined tracking system has momentum resolution $\Delta p / p$ that varies from $0.4 \%$ at $5 \mathrm{GeV} / c$ to $0.6 \%$ at $100 \mathrm{GeV} / c$, 
and impact parameter resolution of $20 \mu \mathrm{m}$ for tracks with high transverse momentum $\left(p_{\mathrm{T}}\right)$. Charged hadrons are identified using two ring-imaging Cherenkov detectors. Photon, electron and hadron candidates are identified by a calorimeter system consisting of scintillatingpad and pre-shower detectors, an electromagnetic calorimeter and a hadronic calorimeter. Muons are identified by a system composed of alternating layers of iron and multiwire proportional chambers. The trigger [32] consists of a hardware stage, based on information from the calorimeter and muon systems, followed by a software stage that applies a full event reconstruction. Samples of open charm mesons produced directly in the primary $p p$ interaction (refered to as 'prompt') and in semileptonic decays of $b$-hadrons are selected by the trigger. Though the prompt sample is larger in size, cuts on the decay time of the $D$ meson are applied at the trigger level to reduce the output rate. As the reconstructed mass and decay time are correlated, these cuts bias the mass measurement. In contrast, no cuts on the $D$ decay time are applied at the trigger level for the semileptonic sample, which is therefore used for this analysis.

The measurements require the momenta of the final state particles to be determined accurately. The procedure used to calibrate the momentum scale of the tracking system for this study is discussed in detail in ref. [33]. It is based upon large calibration samples of $B^{+} \rightarrow J / \psi K^{+}$and $J / \psi \rightarrow \mu^{+} \mu^{-}$decays collected concurrently with the dataset used for this analysis. The use of the large $J / \psi$ dataset allows to correct for variations of the momentum scale at the level of $10^{-4}$ or less that occur over the course of the data-taking period whilst the use of the $B^{+} \rightarrow J / \psi K^{+}$allows the momentum scale to be determined as a function of the $K^{+}$kinematics. The accuracy of the procedure has been checked using other fully reconstructed $B$ decays together with two-body $\Upsilon(n S)$ and $K_{\mathrm{S}}^{0}$ decays. In each case the deviation of the measured mass from the expected value is converted to an estimate of the bias on the momentum scale $(\alpha)$ taking into account relativistic kinematics and QED radiative corrections. The largest value of $|\alpha|$ found in these studies is $0.03 \%$ for the $K_{\mathrm{S}}^{0} \rightarrow \pi^{+} \pi^{-}$decay mode. Conservatively, this is taken as the uncertainty on the calibrated momentum scale. This leads to the largest contribution to the systematic uncertainty on the mass measurements.

\section{Selection}

The selection uses only well reconstructed charged particles that traverse the entire tracking system. All charged particles are required to be within the angular acceptance of the spectrometer. This corresponds to $300 \mathrm{mrad}$ in the bending plane of the dipole magnet and $250 \mathrm{mrad}$ in the orthogonal plane. In addition, the final state particles are required to have $p_{\mathrm{T}}$ greater than $300 \mathrm{MeV} / c$. Further background suppression is achieved by exploiting the fact that the products of heavy flavour decays have a large distance of closest approach ('impact parameter') with respect to the $p p$ interaction vertex in which they were produced. The impact parameter $\chi^{2}$ with respect to any primary vertex is required to be larger than nine. Fake tracks created by the reconstruction are suppressed by cutting on the output of a neural network trained to discriminate between these and real particles. This cut also removes candidates where one of the charged hadrons has decayed in flight. To select 
well-identified kaons (pions) the difference in the logarithms of the global likelihood of the kaon (pion) hypothesis relative to the pion (kaon) hypothesis provided by the ring-imaging Cherenkov detectors is required to be greater than five (zero).

Charged particles selected in this way are combined to form $D^{0} \rightarrow K^{+} K^{-} \pi^{+} \pi^{-}$, $D^{0} \rightarrow K^{+} K^{-} K^{-} \pi^{+}$and $D_{(s)}^{+} \rightarrow K^{+} K^{-} \pi^{+}$candidates. To eliminate kinematic reflections due to misidentified pions, the invariant mass of at least one kaon pair is required to be within $\pm 12 \mathrm{MeV} / \mathrm{c}^{2}$ of the nominal value of the $\phi$ meson mass [4]. This requirement means that the $D$ meson sample is dominated by decays containing an intermediate $\phi$ meson. A fit requiring the final state particles to originate from a common point is made and the $\chi^{2}$ per degree of freedom $\left(\chi^{2} / \mathrm{ndf}\right)$ of this fit is required to be less than five. In order to remove poorly reconstructed candidates, a cut is made on the uncertainty of the reconstructed invariant mass estimated by propagation of the individual track covariance matrices. The value of this cut depends on the decay mode under consideration and is chosen such that the bulk of the distribution is kept and only events in the tail are rejected. In a few percent of the events the reconstruction procedure gives rise to duplicate candidates. Therefore, if two or more candidates that are separated by less than 0.05 in pseudorapidity and $50 \mathrm{mrad}$ in azimuthal angle are found within one event, only that with the best $D$ vertex $\chi^{2}$ is kept.

Each candidate $D$ meson, selected in this way, is combined with a well-identified muon that is displaced from the $p p$ interaction vertex (impact parameter $\chi^{2}>4$ ) and that has $p_{\mathrm{T}}$ larger than $800 \mathrm{MeV} / c$ to form a $B$ candidate. A fit is made requiring the muon and the $D$ candidate to originate from a common point and the $\chi^{2}$ per degree of freedom of this fit is required to be less than five. To select semileptonic $B$ decays, the invariant mass of the $B$ candidate is required to be in the range $2.5-6.0 \mathrm{GeV} / c^{2}$. In principle, the large combinatorial background can be further reduced by cutting on the decay time of the $D$ meson, but due to the correlation between the decay time and the mass, this cut would bias the mass distribution. Therefore, a cut requiring significant displacement between the $b$-hadron decay vertex and the associated $p p$ interaction vertex is applied. This achieves high signal purity whilst not biasing the distribution of the $D$ decay time.

\section{Fit results}

The $D$ meson masses are determined by performing extended unbinned maximum likelihood fits to the invariant mass distributions. In these fits the background is modelled by an exponential function and the signal by the sum of a Crystal Ball [34] and a Gaussian function. The Crystal Ball component accounts for the presence of the QED radiative tail. Alternative models for both the signal and background components are considered as part of the studies of the systematic uncertainties. The model for the signal shape contains six parameters:

- a common mean value for the Gaussian and Crystal Ball components;

- the widths of the Gaussian $\left(\sigma_{\mathrm{G}}\right)$ and the Crystal Ball $\left(\sigma_{\mathrm{CB}}\right)$ components;

- the transition point $(a)$ and exponent $(n)$ of the Crystal Ball component;

- the relative fraction of the Crystal Ball $\left(f_{\mathrm{CB}}\right)$ component. 

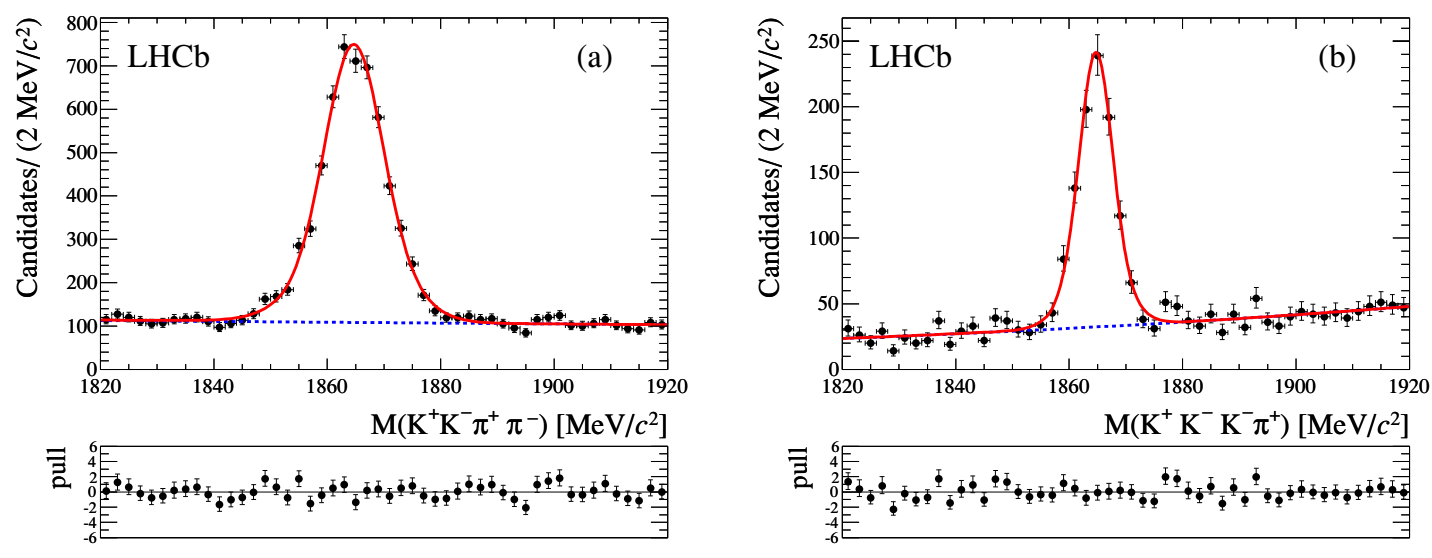

Figure 1. Invariant mass distributions for the (a) $K^{+} K^{-} \pi^{+} \pi^{-}$and (b) $K^{+} K^{-} K^{-} \pi^{+}$final states. In each case the result of the fit described in the text is superimposed (solid line) together with the background component (dotted line). The pull, i.e. the difference between the fitted and measured value divided by the uncertainty on the measured value, is shown below each plot.

To reduce the number of free parameters in the fit, $a, n$ and $f_{\mathrm{CB}}$ together with the ratio of $\sigma_{\mathrm{CB}}$ to $\sigma_{\mathrm{G}}$, are fixed using a simulation that has been tuned to reproduce the mass resolution observed in data for the $B^{+} \rightarrow J / \psi K^{+}$and $B^{+} \rightarrow J / \psi K^{+} \pi^{-} \pi^{+}$decay modes. By fixing the ratio of $\sigma_{\mathrm{CB}}$ to $\sigma_{\mathrm{G}}$ the resolution model is constrained up to an overall resolution scale factor that is close to unity.

The Crystal Ball function describes the effect of the radiative tail far from the peak well. However, close to the peak its shape is still Gaussian, which results in a bias on the fitted mass that scales with the $Q$-value of the decay mode. This effect is studied using Рнотоs [27] to model the effect of QED radiative corrections. The size of the bias is found to be $0.03 \pm 0.01 \mathrm{MeV} / c^{2}$ for the $D^{0} \rightarrow K^{+} K^{-} K^{-} \pi^{+}$mode. For the $D^{0} \rightarrow K^{+} K^{-} \pi^{+} \pi^{-}$, $D^{+} \rightarrow K^{+} K^{-} \pi^{+}$and $D_{s}^{+} \rightarrow K^{+} K^{-} \pi^{+}$decay modes a value of $0.06 \pm 0.01 \mathrm{MeV} / c^{2}$ is found. These values are used to correct the mass measurements. The effect cancels in the measurement of the mass differences.

The resulting fits for the $D^{0}$ decay modes are shown in figure 1 and that for the $K^{+} K^{-} \pi^{+}$final state in figure 2 . The values obtained in these fits are summarized in table 1 . The resulting values of the $D^{+}$and $D_{s}^{+}$masses are in agreement with the current world averages. These modes have relatively large $Q$-values and consequently the systematic uncertainty due to the knowledge of the momentum scale is at the level of $0.3 \mathrm{MeV} / c^{2}$. Hence, it is chosen not to quote these values as measurements. Similarly, the systematic uncertainty due to the momentum scale for the $D^{0} \rightarrow K^{+} K^{-} \pi^{+} \pi^{-}$mode is estimated to be $0.2 \mathrm{MeV} / c^{2}$ and the measured mass in this mode is not used in the $D^{0}$ mass determination.

The quality of the fits is judged from the $\chi^{2} /$ ndf, quoted in table 1 , and the fit residuals. It has been checked using simulated pseudo-experiments that the sizeable trends seen in the residuals for the $K^{+} K^{-} \pi^{+}$mode, where the dataset is largest, do not bias the mass difference measurement. The fitted resolution scale factors are all within a few percent of unity, indicating that the calibration parameters obtained from the $B^{+}$study are applicable 


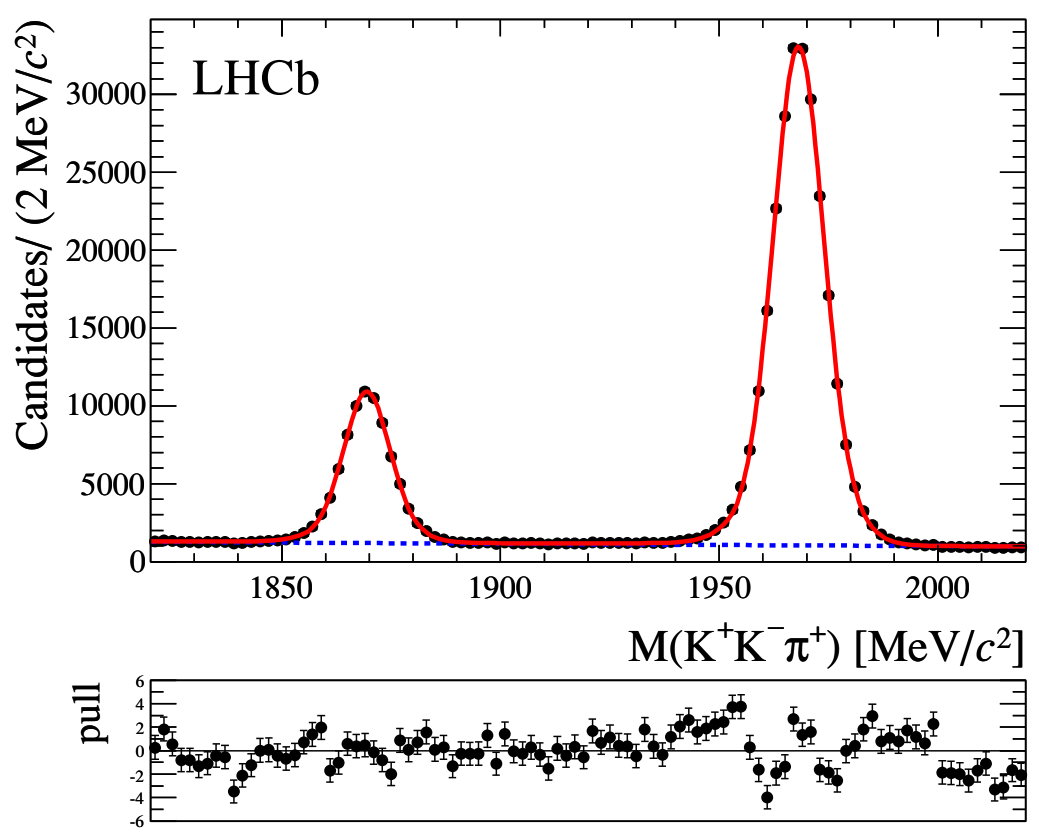

Figure 2. Invariant mass distribution for the $K^{+} K^{-} \pi^{+}$final state. The result of the fit described in the text is superimposed (solid line) together with the background component (dotted line). The pull, i.e. the difference between the fitted value and the measured value divided by the uncertainty, is shown below the plot.

\begin{tabular}{|l|c|c|c|c|c|}
\hline Decay mode & Yield & $\begin{array}{c}\text { Fitted mass } \\
{\left[\mathrm{MeV} / c^{2}\right]}\end{array}$ & $\begin{array}{c}\text { Corrected mass } \\
{\left[\mathrm{MeV} / c^{2}\right]}\end{array}$ & $\begin{array}{c}\text { Resolution } \\
\text { scale factor }\end{array}$ & $\chi^{2} / \mathrm{ndf}$ \\
\hline$D^{0} \rightarrow K^{+} K^{-} \pi^{+} \pi^{-}$ & $4608 \pm 89$ & $1864.68 \pm 0.12$ & $1864.74 \pm 0.12$ & $1.031 \pm 0.021$ & 0.83 \\
$D^{0} \rightarrow K^{+} K^{-} K^{-} \pi^{+}$ & $849 \pm 36$ & $1864.73 \pm 0.15$ & $1864.75 \pm 0.15$ & $0.981 \pm 0.042$ & 0.92 \\
$D^{+} \rightarrow K^{+} K^{-} \pi^{+}$ & $68,787 \pm 321$ & $1869.44 \pm 0.03$ & $1869.50 \pm 0.03$ & $0.972 \pm 0.003$ & 2.5 \\
$D_{s}^{+} \rightarrow K^{+} K^{-} \pi^{+}$ & $248,694 \pm 540$ & $1968.13 \pm 0.03$ & $1968.19 \pm 0.03$ & $0.971 \pm 0.002$ & \\
\hline
\end{tabular}

Table 1. Signal yields, mass values, resolution scale factors and binned $\chi^{2} / \mathrm{ndf}$ (using 100 bins) obtained from the fits shown in figure 1 and figure 2 together with the values corrected for the effect of QED radiative corrections as described in the text.

in this analysis. The uncertainties on the masses reported by the fits are in good agreement with the results obtained in pseudo-experiments.

Using the values in table 1 , the mass differences are evaluated to be

$$
\begin{aligned}
& M\left(D^{+}\right)-M\left(D^{0}\right)=4.76 \pm 0.12(\text { stat }) \mathrm{MeV} / c^{2} \\
& M\left(D_{s}^{+}\right)-M\left(D^{+}\right)=98.68 \pm 0.03(\text { stat }) \mathrm{MeV} / c^{2}
\end{aligned}
$$

where the uncertainties are statistical only. 


\begin{tabular}{|l|r|c|c|}
\hline Source of uncertainty & $M\left(D^{0}\right)$ & $M\left(D^{+}\right)-M\left(D^{0}\right)$ & $M\left(D_{s}^{+}\right)-M\left(D^{+}\right)$ \\
\hline Momentum scale & 0.09 & 0.04 & 0.04 \\
Energy loss correction & 0.03 & 0.06 & $<0.01$ \\
$K^{ \pm}$mass & 0.05 & $<0.01$ & $<0.01$ \\
Signal model & 0.02 & $<0.01$ & $<0.01$ \\
Background model & $<0.01$ & $<0.01$ & $<0.01$ \\
\hline Quadratic sum & 0.11 & 0.07 & 0.04 \\
\hline
\end{tabular}

Table 2. Systematic uncertainties (in $\mathrm{MeV} / \mathrm{c}^{2}$ ) on the mass measurements and on their differences.

\section{$5 \quad$ Systematic uncertainties}

To evaluate the systematic uncertainty, the complete analysis is repeated, including the track fit and the momentum scale calibration when needed, varying within their uncertainties the parameters to which the mass determination is sensitive. The observed changes in the central values of the fitted masses relative to the nominal results are assigned as systematic uncertainties.

The dominant source of uncertainty is the limited knowledge of the momentum scale. The mass fits are repeated with the momentum scale varied by $\pm 0.03 \%$. A further uncertainty is related to the understanding of the energy loss in the material of the tracking system. The amount of material traversed in the tracking system by a particle is known to $10 \%$ accuracy [35]. Therefore, the magnitude of the energy loss correction in the reconstruction is varied by $\pm 10 \%$.

Other uncertainties arise from the fit model. To evaluate the impact of the signal model, a fit is performed where all signal parameters are fixed according to the values found in the simulation and a second fit where the parameters $\sigma_{\mathrm{G}}$ and $\sigma_{\mathrm{CB}}$ are allowed to vary while keeping the relative fraction, $f_{\mathrm{CB}}$, of the two components fixed. The larger of the differences to the default fit result is assigned as an estimate of the systematic uncertainty. Similarly the effect of the background modelling is estimated by replacing the exponential function with a first-order Chebychev polynomial. The shifts of the mass values observed in these tests are generally much smaller than $0.01 \mathrm{MeV} / c^{2}$ and are assigned as systematic uncertainties. For the $K^{+} K^{-} \pi^{+}$fit further cancellation occurs in the mass difference. It is concluded that the details of the fit model have little effect on the presented measurements.

An additional uncertainty arises from the knowledge of the value of the $K^{+}$mass, $m_{K^{ \pm}}=493.677 \pm 0.016 \mathrm{MeV} / c^{2}$ [4]. The effect of this uncertainty on the measurements has been evaluated from simulation studies.

The systematic uncertainties on the measured masses and mass differences are summarized in table 2. The uncertainties related to the momentum scale and energy loss correction are fully correlated between the measurements.

Various cross-checks of the measurements are made. Two checks are related to the knowledge of the tracking system alignment. First, a study has been performed where particle trajectories are reconstructed without using the information related to the tracking detector located before the entrance of the spectrometer magnet. This information is not 
required to form a track but improves the momentum resolution by $10-20 \%$. The second test is to vary the track slopes in the vertex detector by the uncertainty of $2 \times 10^{-4}$ on the length scale of the detector described in ref. [36]. The results obtained in these studies are consistent with those presented here and no additional uncertainty is assigned.

A further check for the $D^{0}$ mass measurement is the comparison of the measured mass in the $D^{0} \rightarrow K^{+} K^{-} K^{-} \pi^{+}$mode with that obtained in the three other four-body modes. Systematic effects related to the momentum scale will affect modes with a high $Q$-value more than those with low $Q$-values. The relationship between the reconstructed mass $(m)$ and the momentum scale $(\alpha)$ after a first-order Taylor expansion in $m^{2} / p^{2}$ is

$$
m^{2}=\frac{m_{\text {true }}^{2}-f}{(1-\alpha)^{2}}+f
$$

where

$$
f=p \sum \frac{m_{i}^{2}}{p_{i}}
$$

$p$ is the total momentum of the decaying meson and $p_{i}$ and $m_{i}$ are the momenta and masses of the daughter particles. This formalism assumes that there are no additional differences affecting the momentum scale between the modes such as differences in track kinematics or the effect of QED radiative corrections. For each decay mode the average value of $f$ is obtained from the data using the sPlot technique [37] with the mass as the control variable to subtract the effect of background. The values obtained in this way are in good agreement with those found in the simulation. In figure 3 the measured $D^{0}$ mass is plotted versus $f$ for the four-body decay modes studied here. The shaded area on this plot corresponds to the assigned systematic uncertainty of $0.03 \%$ on the momentum scale. Though there is evidence of a systematic effect for the low $f$-value modes it is accounted for by the assigned uncertainty.

The dataset has also been divided according to the magnet polarity and data-taking period and for the charged modes by the sign of the product of the magnet polarity and the $D$ meson charge. In addition, for modes where the event samples are sizable the measurements are repeated in bins of the $D$ meson kinematic variables. None of these tests reveal any evidence of a systematic bias.

\section{Summary}

Measurements of $D$ meson masses and mass differences have been performed using $p p$ collision data, corresponding to an integrated luminosity of $1.0 \mathrm{fb}^{-1}$ collected at a centreof-mass energy of $\sqrt{s}=7 \mathrm{TeV}$ with the $\mathrm{LHCb}$ detector. The results are

$$
\begin{array}{lrr}
M\left(D^{0}\right) & =1864.75 \pm 0.15 \text { (stat) } \pm 0.11 \text { (syst) } \mathrm{MeV} / c^{2}, \\
M\left(D^{+}\right)-M\left(D^{0}\right)= & 4.76 \pm 0.12 \text { (stat) } \pm 0.07 \text { (syst) } \mathrm{MeV} / c^{2}, \\
M\left(D_{s}^{+}\right)-M\left(D^{+}\right)= & 98.68 \pm 0.03 \text { (stat) } \pm 0.04 \text { (syst) } \mathrm{MeV} / c^{2} .
\end{array}
$$

The dominant systematic uncertainty is related to the knowledge of the momentum scale. 


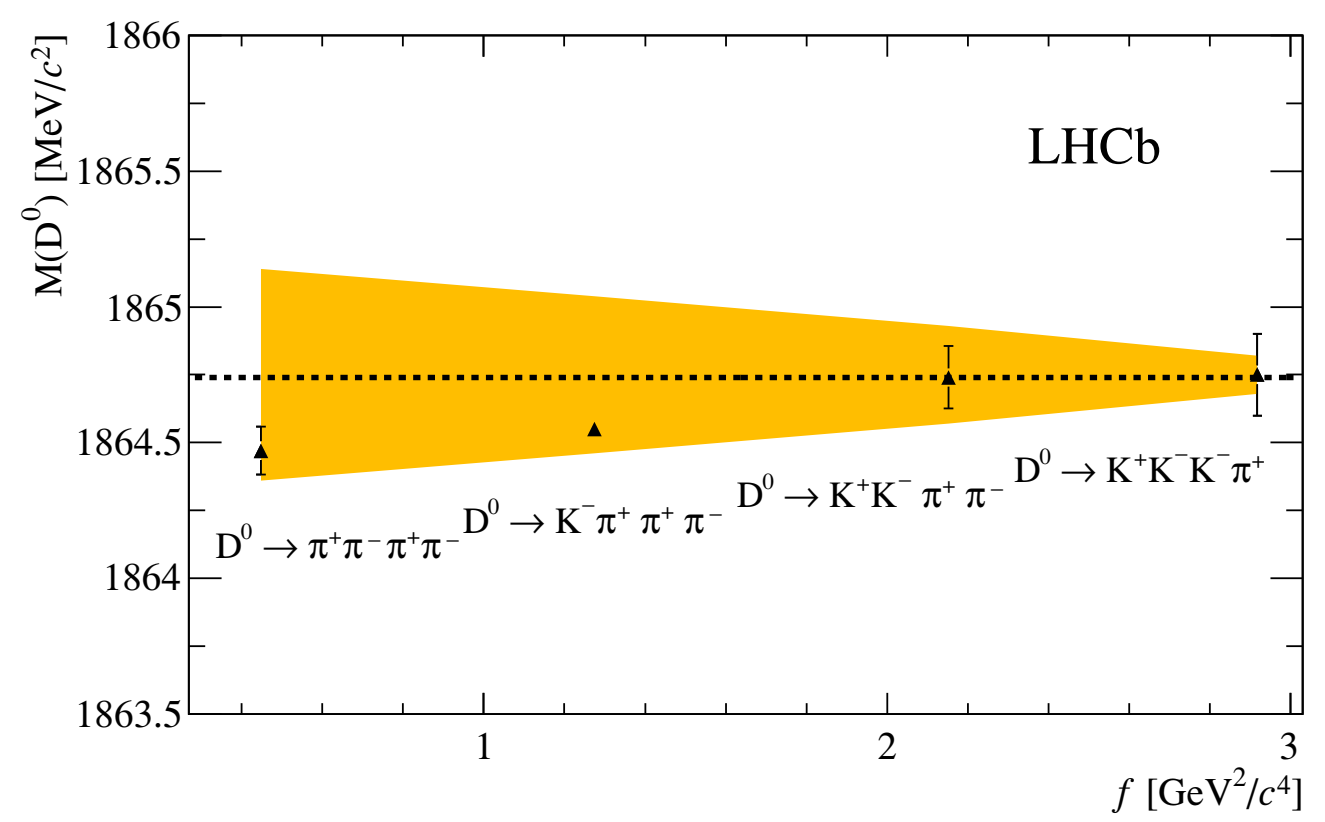

Figure 3. Measured $D^{0}$ mass versus $f$ as defined in eq. (5.2). The (yellow) shaded area corresponds to a systematic uncertainty on the momentum scale of $0.03 \%$ centred on the result for the $D^{0} \rightarrow$ $K^{+} K^{-} K^{-} \pi^{+}$mode (horizontal dashed line). Only the $D^{0} \rightarrow K^{+} K^{-} K^{-} \pi^{+}$mode, where the systematic uncertainty is lowest, is used to determine the $D^{0}$ mass.

\begin{tabular}{|c|r|r|r|}
\hline Quantity & \multicolumn{1}{|c|}{$\begin{array}{c}\text { LHCb } \\
\text { measurement }\end{array}$} & \multicolumn{1}{|c|}{$\begin{array}{c}\text { Best previous } \\
\text { measurement }\end{array}$} & \multicolumn{1}{c|}{ PDG fit [4] } \\
\hline$M\left(D^{0}\right)$ & $1864.75 \pm 0.19$ & $1864.85 \pm 0.18[5]$ & $1864.86 \pm 0.13$ \\
$M\left(D^{+}\right)-M\left(D^{0}\right)$ & $4.76 \pm 0.14$ & $4.7 \pm 0.3 \quad[7]$ & $4.76 \pm 0.10$ \\
$M\left(D_{s}^{+}\right)-M\left(D^{+}\right)$ & $98.68 \pm 0.05$ & $98.4 \pm 0.3 \quad[10]$ & $98.88 \pm 0.25$ \\
\hline
\end{tabular}

Table 3. LHCb measurements, compared to the best previous measurements and to the results of a global fit to available open charm mass data. The quoted uncertainties are the quadratic sums of the statistical and systematic contributions. All values are in $\mathrm{MeV} / \mathrm{c}^{2}$.

As shown in table 3, these measurements are in agreement with previous measurements. The results for the mass differences have smaller uncertainty than any previously reported value. The measured value of the $D^{0}$ mass has a similar precision to the published CLEO result [5]. Including this result in the determination of the $X(3872)$ binding energy given in section 1 gives $E_{B}=0.09 \pm 0.28 \mathrm{MeV} / c^{2}$. This reinforces the conclusion that if the $X(3872)$ state is a molecule it is extremely loosely bound.

The measurements presented here, together with those given in ref. [4] for the $D^{+}$and $D^{0}$ mass, and the mass differences $M\left(D^{+}\right)-M\left(D^{0}\right), M\left(D_{s}^{+}\right)-M\left(D^{+}\right)$can be used to determine a more precise value of the $D_{s}^{+}$mass

$$
M\left(D_{s}^{+}\right)=1968.19 \pm 0.20 \pm 0.14 \pm 0.08 \mathrm{MeV} / c^{2},
$$

where the first uncertainty is the quadratic sum of the statistical and uncorrelated systematic uncertainty, the second is due to the momentum scale and the third due to the energy loss. 
This value is consistent with, but more precise than, that obtained from the fit to open charm mass data, $M\left(D_{s}^{+}\right)=1968.49 \pm 0.32 \mathrm{MeV} / c^{2}[4]$.

\section{Acknowledgments}

We express our gratitude to our colleagues in the CERN accelerator departments for the excellent performance of the LHC. We thank the technical and administrative staff at the LHCb institutes. We acknowledge support from CERN and from the national agencies: CAPES, CNPq, FAPERJ and FINEP (Brazil); NSFC (China); CNRS/IN2P3 and Region Auvergne (France); BMBF, DFG, HGF and MPG (Germany); SFI (Ireland); INFN (Italy); FOM and NWO (The Netherlands); SCSR (Poland); ANCS/IFA (Romania); MinES, Rosatom, RFBR and NRC "Kurchatov Institute" (Russia); MinECo, XuntaGal and GENCAT (Spain); SNSF and SER (Switzerland); NAS Ukraine (Ukraine); STFC (United Kingdom); NSF (U.S.A.). We also acknowledge the support received from the ERC under FP7. The Tier1 computing centres are supported by IN2P3 (France), KIT and BMBF (Germany), INFN (Italy), NWO and SURF (The Netherlands), PIC (Spain), GridPP (United Kingdom). We are thankful for the computing resources put at our disposal by Yandex LLC (Russia), as well as to the communities behind the multiple open source software packages that we depend on.

Open Access. This article is distributed under the terms of the Creative Commons Attribution License which permits any use, distribution and reproduction in any medium, provided the original author(s) and source are credited.

\section{References}

[1] BaBAr collaboration, B. Aubert et al., Measurement of the Mass Difference $M\left(B^{0}\right)-M\left(B^{+}\right)$, Phys. Rev. D 78 (2008) 011103 [arXiv: 0805. 0497] [InSPIRE].

[2] CDF collaboration, D. Acosta et al., Measurement of $b$ hadron masses in exclusive $J / \psi$ decays with the CDF detector, Phys. Rev. Lett. 96 (2006) 202001 [hep-ex/0508022] [INSPIRE].

[3] LHCb collaboration, Measurement of b-hadron masses, Phys. Lett. B 708 (2012) 241 [arXiv:1112.4896] [INSPIRE].

[4] Particle Data Group collaboration, J. Beringer et al., Review of Particle Physics (RPP), Phys. Rev. D 86 (2012) 010001 [INSPIRE].

[5] CLEO collaboration, C. Cawlfield et al., A Precision Determination of the $D^{0}$ Mass, Phys. Rev. Lett. 98 (2007) 092002 [hep-ex/0701016] [INSPIRE].

[6] KEDR collaboration, V. Anashin et al., Measurement of $D^{0}$ and $D^{+}$meson masses with the KEDR Detector, Phys. Lett. B 686 (2010) 84 [arXiv: 0909.5545] [INSPIRE].

[7] R. Schindler, M. Alam, A. Boyarski, M. Breidenbach, D. Burke et al., Measurements of the Properties of D Meson Decays, Phys. Rev. D 24 (1981) 78 [InSPIRE].

[8] I. Peruzzi, M. Piccolo, G. Feldman, P. Lecomte, V. Vuillemin et al., Study of D Mesons Produced in the Decay of the $\psi$ (3772), Phys. Rev. Lett. 39 (1977) 1301 [INSPIRE]. 
[9] CDF collaboration, D. Acosta et al., Measurement of the mass difference $m\left(D_{s}^{+}\right)-m\left(D^{+}\right)$at CDF II, Phys. Rev. D 68 (2003) 072004 [hep-ex/0310043] [INSPIRE].

[10] BABAR collaboration, B. Aubert et al., Measurement of $D_{s}^{+}$and $D_{s}^{*+}$ production in $B$ meson decays and from continuum $e^{+} e^{-}$annihilation at $\sqrt{s}=10.6$ GeV, Phys. Rev. D 65 (2002) 091104 [hep-ex/0201041] [INSPIRE].

[11] CLEO collaboration, D.N. Brown et al., Precision measurement of the $D_{(s)}^{*+}-D_{(s)}^{+}$mass difference, Phys. Rev. D 50 (1994) 1884 [hep-ph/9403327] [INSPIRE].

[12] CLEO collaboration, W. Chen et al., Measurement of $D_{s}$ decay modes, Phys. Lett. B 226 (1989) 192 [INSPIRE].

[13] J. Anjos, J. Appel, A. Bean, S.B. Bracker, T. Browder et al., Measurement of $D_{X}^{ \pm}$Decays and Cabibbo Suppressed $D^{ \pm}$Decays, Phys. Rev. Lett. 60 (1988) 897 [INSPIRE].

[14] LHCb collaboration, Observation of $B_{c}^{+} \rightarrow J / \psi D_{s}^{+}$and $B_{c}^{+} \rightarrow J / \psi D_{s}^{*+}$ decays, arXiv: 1304.4530 [INSPIRE].

[15] Belle collaboration, S. Choi et al., Observation of a narrow charmonium - like state in exclusive $B^{ \pm} \rightarrow K^{ \pm} \pi^{+} \pi^{-} J / \psi$ decays, Phys. Rev. Lett. 91 (2003) 262001 [hep-ex/0309032] [INSPIRE].

[16] CDF collaboration, D. Acosta et al., Observation of the narrow state $X(3872) \rightarrow J / \psi \pi^{+} \pi^{-}$in $\bar{p} p$ collisions at $\sqrt{s}=1.96 \mathrm{TeV}$, Phys. Rev. Lett. 93 (2004) 072001 [hep-ex/0312021] [INSPIRE].

[17] D0 collaboration, V. Abazov et al., Observation and properties of the X(3872) decaying to $J / \psi \pi^{+} \pi^{-}$in pp collisions at $\sqrt{s}=1.96$ TeV, Phys. Rev. Lett. 93 (2004) 162002 [hep-ex/0405004] [INSPIRE].

[18] BABAR collaboration, B. Aubert et al., Study of the $B \rightarrow J / \psi K^{-} \pi^{+} \pi^{-}$decay and measurement of the $B \rightarrow X(3872) K^{-}$branching fraction, Phys. Rev. D 71 (2005) 071103 [hep-ex/0406022] [INSPIRE].

[19] LHCb collaboration, Observation of $X(3872)$ production in pp collisions at $\sqrt{s}=7 \mathrm{TeV}$, Eur. Phys. J. C 72 (2012) 1972 [arXiv:1112.5310] [INSPIRE].

[20] CMS collaboration, Measurement of the X(3872) production cross section via decays to $J / \psi \pi^{+} \pi^{-}$in pp collisions at $\sqrt{s}=7 \mathrm{TeV}$, JHEP 04 (2013) 154 [arXiv: 1302.3968] [INSPIRE].

[21] LHCb collaboration, Determination of the X(3872) meson quantum numbers, Phys. Rev. Lett. 110 (2013) 222001 [arXiv: 1302.6269] [INSPIRE].

[22] L. Maiani, F. Piccinini, A. Polosa and V. Riquer, Diquark-antidiquarks with hidden or open charm and the nature of X(3872), Phys. Rev. D 71 (2005) 014028 [hep-ph/0412098] [INSPIRE].

[23] N.A. Tornqvist, Isospin breaking of the narrow charmonium state of Belle at $3872 \mathrm{MeV}$ as a deuson, Phys. Lett. B 590 (2004) 209 [hep-ph/0402237] [INSPIRE].

[24] T. Sjöstrand, S. Mrenna and P.Z. Skands, PYTHIA 6.4 Physics and Manual, JHEP 05 (2006) 026 [hep-ph/0603175] [inSPIRE].

[25] I. Belyaev et al., Handling of the generation of primary events in GAUss, the LHCb simulation framework, Nuclear Science Symposium Conference Record (NSS/MIC) IEEE (2010) 1155.

[26] D. Lange, The EvtGen particle decay simulation package, Nucl. Instrum. Meth. A 462 (2001) 152 [INSPIRE]. 
[27] P. Golonka and Z. Was, PHOTOS Monte Carlo: A Precision tool for QED corrections in Z and $W$ decays, Eur. Phys. J. C 45 (2006) 97 [hep-ph/0506026] [INSPIRE].

[28] GEANT4 collaboration, J. Allison et al., Geant4 developments and applications, IEEE Trans. Nucl. Sci. 53 (2006) 270.

[29] GEANT4 collaboration, S. Agostinelli et al., GEANT4: A Simulation toolkit, Nucl. Instrum. Meth. A 506 (2003) 250 [INSPIRE].

[30] M. Clemencic et al., The LHCb simulation application, GAUss: design, evolution and experience, J. Phys. Conf. Ser. 331 (2011) 032023 [InSPIRE].

[31] LHCb collaboration, The LHCb Detector at the LHC, 2008 JINST 3 S08005 [InSPIRE].

[32] R. Aaij, J. Albrecht, F. Alessio, S. Amato, E. Aslanides et al., The LHCb Trigger and its Performance in 2011, 2013 JINST 8 P04022 [arXiv:1211.3055] [INSPIRE].

[33] LHCb collaboration, Measurement of the $\Lambda_{b}^{0}, \Xi_{b}^{-}$and $\Omega_{b}^{-}$baryon masses, Phys. Rev. Lett. 110 (2013) 182001 [arXiv:1302.1072] [INSPIRE].

[34] T. Skwarnicki, A study of the radiative cascade transitions between the $\Upsilon$-prime and $\Upsilon$ resonances, Ph.D. Thesis, Institute of Nuclear Physics, Krakow (1986) [INSPIRE].

[35] LHCb collaboration, Prompt $K_{s}^{0}$ production in pp collisions at $\sqrt{s}=0.9 \mathrm{TeV}$, Phys. Lett. $\mathbf{B}$ 693 (2010) 69 [arXiv: 1008.3105] [inSPIRE].

[36] LHCb collaboration, Precision measurement of the $B_{s}^{0}-\bar{B}_{s}^{0}$ oscillation frequency with the decay $B_{s}^{0} \rightarrow D_{s}^{-} \pi^{+}$, New J. Phys. 15 (2013) 053021 [arXiv:1304.4741] [InSPIRE].

[37] M. Pivk and F.R. Le Diberder, SPlot: A Statistical tool to unfold data distributions, Nucl. Instrum. Meth. A 555 (2005) 356 [physics/0402083] [INSPIRE]. 


\section{The LHCb collaboration}

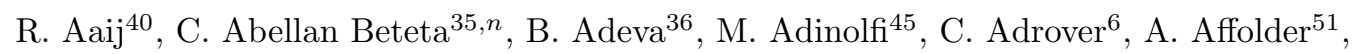
Z. Ajaltouni ${ }^{5}$, J. Albrecht ${ }^{9}$, F. Alessio ${ }^{37}$, M. Alexander ${ }^{50}$, S. Ali ${ }^{40}$, G. Alkhazov ${ }^{29}$, P. Alvarez Cartelle ${ }^{36}$, A.A. Alves $\mathrm{Jr}^{24,37}$, S. Amato ${ }^{2}$, S. Amerio ${ }^{21}$, Y. Amhis ${ }^{7}$, L. Anderlini ${ }^{17, f}$, J. Anderson ${ }^{39}$, R. Andreassen ${ }^{56}$, R.B. Appleby ${ }^{53}$, O. Aquines Gutierrez ${ }^{10}$, F. Archilli ${ }^{18}$, A. Artamonov ${ }^{34}$, M. Artuso ${ }^{57}$, E. Aslanides ${ }^{6}$, G. Auriemma ${ }^{24, m}$, S. Bachmann ${ }^{11}$, J.J. Back ${ }^{47}$, C. Baesso ${ }^{58}$, V. Balagura ${ }^{30}$, W. Baldini ${ }^{16}$, R.J. Barlow ${ }^{53}$, C. Barschel ${ }^{37}$, S. Barsuk ${ }^{7}$, W. Barter ${ }^{46}$, Th. Bauer ${ }^{40}$, A. Bay ${ }^{38}$, J. Beddow ${ }^{50}$, F. Bedeschi ${ }^{22}$, I. Bediaga ${ }^{1}$, S. Belogurov ${ }^{30}$, K. Belous ${ }^{34}$, I. Belyaev ${ }^{30}$, E. Ben-Haim ${ }^{8}$, M. Benayoun ${ }^{8}$, G. Bencivenni ${ }^{18}$, S. Benson ${ }^{49}$, J. Benton ${ }^{45}$, A. Berezhnoy ${ }^{31}$, R. Bernet ${ }^{39}$, M.-O. Bettler ${ }^{46}$, M. van Beuzekom ${ }^{40}$, A. Bien ${ }^{11}$, S. Bifani ${ }^{44}$, T. Bird ${ }^{53}$, A. Bizzeti ${ }^{17, h}$, P.M. Bjørnstad ${ }^{53}$, T. Blake ${ }^{37}$, F. Blanc ${ }^{38}$, J. Blouw ${ }^{11}$, S. Blusk ${ }^{57}$, V. Bocci ${ }^{24}$, A. Bondar ${ }^{33}$, N. Bondar ${ }^{29}$, W. Bonivento ${ }^{15}$, S. Borghi ${ }^{53}$, A. Borgia ${ }^{57}$, T.J.V. Bowcock ${ }^{51}$, E. Bowen ${ }^{39}$, C. Bozzi ${ }^{16}$, T. Brambach ${ }^{9}$, J. van den Brand ${ }^{41}$, J. Bressieux ${ }^{38}$, D. Brett ${ }^{53}$, M. Britsch ${ }^{10}$, T. Britton ${ }^{57}$, N.H. Brook ${ }^{45}$, H. Brown ${ }^{51}$, I. Burducea ${ }^{28}$, A. Bursche ${ }^{39}$, G. Busetto ${ }^{21, q}$, J. Buytaert ${ }^{37}$, S. Cadeddu ${ }^{15}$, O. Callot ${ }^{7}$, M. Calvi ${ }^{20, j}$, M. Calvo Gomez ${ }^{35, n}$, A. Camboni ${ }^{35}$, P. Campana ${ }^{18,37}$, D. Campora Perez ${ }^{37}$, A. Carbone ${ }^{14, c}$, G. Carboni ${ }^{23, k}$, R. Cardinale ${ }^{19, i}$, A. Cardini ${ }^{15}$, H. Carranza-Mejia ${ }^{49}$, L. Carson ${ }^{52}$, K. Carvalho Akiba ${ }^{2}$, G. Casse ${ }^{51}$, M. Cattaneo ${ }^{37}$, Ch. Cauet ${ }^{9}$, M. Charles ${ }^{54}$, Ph. Charpentier $^{37}$, P. Chen $^{3,38}$, N. Chiapolini ${ }^{39}$, M. Chrzaszcz ${ }^{25}$, K. Ciba ${ }^{37}$, X. Cid Vidal ${ }^{37}$, G. Ciezarek ${ }^{52}$, P.E.L. Clarke ${ }^{49}$, M. Clemencic ${ }^{37}$, H.V. Cliff ${ }^{46}$, J. Closier ${ }^{37}$, C. Coca ${ }^{28}$, V. Coco $^{40}$, J. Cogan $^{6}$, E. Cogneras ${ }^{5}$, P. Collins ${ }^{37}$, A. Comerma-Montells ${ }^{35}$, A. Contu ${ }^{15,37}$, A. Cook ${ }^{45}$, M. Coombes ${ }^{45}$, S. Coquereau $^{8}$, G. Corti $^{37}$, B. Couturier ${ }^{37}$, G.A. Cowan $^{49}$, D.C. Craik $^{47}$, S. Cunliffe ${ }^{52}$, R. Currie ${ }^{49}$, C. D'Ambrosio ${ }^{37}$, P. David ${ }^{8}$, P.N.Y. David ${ }^{40}$, A. Davis ${ }^{56}$, I. De Bonis ${ }^{4}$, K. De Bruyn ${ }^{40}$, S. De Capua ${ }^{53}$, M. De Cian ${ }^{39}$, J.M. De Miranda ${ }^{1}$, L. De Paula ${ }^{2}$, W. De Silva ${ }^{56}$, P. De Simone ${ }^{18}$, D. Decamp ${ }^{4}$, M. Deckenhoff ${ }^{9}$, L. Del Buono ${ }^{8}$, D. Derkach ${ }^{14}$, O. Deschamps ${ }^{5}$, F. Dettori ${ }^{41}$, A. Di Canto ${ }^{11}$, H. Dijkstra ${ }^{37}$, M. Dogaru ${ }^{28}$, S. Donleavy ${ }^{51}$, F. Dordei ${ }^{11}$, A. Dosil Suárez ${ }^{36}$, D. Dossett ${ }^{47}$, A. Dovbnya ${ }^{42}$, F. Dupertuis ${ }^{38}$, R. Dzhelyadin ${ }^{34}$, A. Dziurda ${ }^{25}$, A. Dzyuba ${ }^{29}$, S. Easo ${ }^{48,37}$, U. Egede ${ }^{52}$, V. Egorychev ${ }^{30}$, S. Eidelman ${ }^{33}$, D. van Eijk ${ }^{40}$, S. Eisenhardt ${ }^{49}$, U. Eitschberger ${ }^{9}$, R. Ekelhof ${ }^{9}$, L. Eklund ${ }^{50,37}$, I. El Rifai ${ }^{5}$, Ch. Elsasser ${ }^{39}$, D. Elsby ${ }^{44}$, A. Falabella ${ }^{14, e}$, C. Färber ${ }^{11}$, G. Fardell ${ }^{49}$, C. Farinelli ${ }^{40}$, S. Farry ${ }^{12}$, V. Fave ${ }^{38}$, D. Ferguson ${ }^{49}$, V. Fernandez Albor ${ }^{36}$, F. Ferreira Rodrigues ${ }^{1}$, M. Ferro-Luzzi ${ }^{37}$, S. Filippov ${ }^{32}$, M. Fiore ${ }^{16}$, C. Fitzpatrick ${ }^{37}$, M. Fontana ${ }^{10}$, F. Fontanelli ${ }^{19, i}$, R. Forty ${ }^{37}$, O. Francisco ${ }^{2}$, M. Frank ${ }^{37}$, C. Frei ${ }^{37}$, M. Frosini ${ }^{17, f}$, S. Furcas ${ }^{20}$, E. Furfaro ${ }^{23, k}$, A. Gallas Torreira ${ }^{36}$, D. Galli ${ }^{14, c}$, M. Gandelman ${ }^{2}$, P. Gandini ${ }^{57}$, Y. Gao ${ }^{3}$, J. Garofoli ${ }^{57}$, P. Garosi ${ }^{53}$, J. Garra Tico ${ }^{46}$, L. Garrido ${ }^{35}$, C. Gaspar ${ }^{37}$, R. Gauld ${ }^{54}$, E. Gersabeck ${ }^{11}$,

M. Gersabeck ${ }^{53}$, T. Gershon ${ }^{47,37}$, Ph. Ghez ${ }^{4}$, V. Gibson ${ }^{46}$, V.V. Gligorov ${ }^{37}$, C. Göbel ${ }^{58}$, D. Golubkov ${ }^{30}$, A. Golutvin ${ }^{52,30,37}$, A. Gomes ${ }^{2}$, H. Gordon ${ }^{54}$, M. Grabalosa Gándara ${ }^{5}$, R. Graciani Diaz ${ }^{35}$, L.A. Granado Cardoso ${ }^{37}$, E. Graugés ${ }^{35}$, G. Graziani ${ }^{17}$, A. Grecu ${ }^{28}$, E. Greening ${ }^{54}$, S. Gregson ${ }^{46}$, O. Grünberg ${ }^{59}$, B. Gui ${ }^{57}$, E. Gushchin ${ }^{32}$, Yu. Guz ${ }^{34,37}$, T. Gys ${ }^{37}$, C. Hadjivasiliou ${ }^{57}$, G. Haefeli ${ }^{38}$, C. Haen ${ }^{37}$, S.C. Haines ${ }^{46}$, S. Hall ${ }^{52}$, T. Hampson ${ }^{45}$, S. Hansmann-Menzemer ${ }^{11}$, N. Harnew ${ }^{54}$, S.T. Harnew ${ }^{45}$, J. Harrison ${ }^{53}$, T. Hartmann ${ }^{59}$, J. He ${ }^{37}$, V. Heijne ${ }^{40}$, K. Hennessy ${ }^{51}$, P. Henrard ${ }^{5}$, J.A. Hernando Morata ${ }^{36}$, E. van Herwijnen ${ }^{37}$, E. Hicks ${ }^{51}$, D. Hill ${ }^{54}$, M. Hoballah ${ }^{5}$, C. Hombach ${ }^{53}$, P. Hopchev ${ }^{4}$, W. Hulsbergen ${ }^{40}$, P. Hunt ${ }^{54}$, T. Huse ${ }^{51}$,

N. Hussain ${ }^{54}$, D. Hutchcroft ${ }^{51}$, D. Hynds ${ }^{50}$, V. Iakovenko ${ }^{43}$, M. Idzik ${ }^{26}$, P. Ilten ${ }^{12}$, R. Jacobsson ${ }^{37}$, A. Jaeger ${ }^{11}$, E. Jans ${ }^{40}$, P. Jaton ${ }^{38}$, F. Jing ${ }^{3}$, M. John ${ }^{54}$, D. Johnson ${ }^{54}$, C.R. Jones ${ }^{46}$, B. Jost ${ }^{37}$, M. Kaballo ${ }^{9}$, S. Kandybei ${ }^{42}$, M. Karacson ${ }^{37}$, T.M. Karbach ${ }^{37}$, I.R. Kenyon ${ }^{44}$, U. Kerzel ${ }^{37}$, T. Ketel ${ }^{41}$, A. Keune ${ }^{38}$, B. Khanji ${ }^{20}$, O. Kochebina ${ }^{7}$, I. Komarov ${ }^{38}$, R.F. Koopman ${ }^{41}$, P. Koppenburg ${ }^{40}$, M. Korolev ${ }^{31}$, A. Kozlinskiy ${ }^{40}$, L. Kravchuk ${ }^{32}$, K. Kreplin ${ }^{11}$, M. Kreps ${ }^{47}$, 
G. Krocker ${ }^{11}$, P. Krokovny ${ }^{33}$, F. Kruse ${ }^{9}$, M. Kucharczyk ${ }^{20,25, j}$, V. Kudryavtsev ${ }^{33}$, T. Kvaratskheliya ${ }^{30,37}$, V.N. La Thi ${ }^{38}$, D. Lacarrere ${ }^{37}$, G. Lafferty ${ }^{53}$, A. Lai ${ }^{15}$, D. Lambert ${ }^{49}$, R.W. Lambert ${ }^{41}$, E. Lanciotti ${ }^{37}$, G. Lanfranchi ${ }^{18}$, C. Langenbruch ${ }^{37}$, T. Latham ${ }^{47}$, C. Lazzeroni ${ }^{44}$, R. Le Gac ${ }^{6}$, J. van Leerdam ${ }^{40}$, J.-P. Lees ${ }^{4}$, R. Lefèvre ${ }^{5}$, A. Leflat ${ }^{31}$, J. Lefrançois ${ }^{7}$, S. Leo $^{22}$, O. Leroy $^{6}$, T. Lesiak ${ }^{25}$, B. Leverington ${ }^{11}$, Y. Li ${ }^{3}$, L. Li Gioi ${ }^{5}$, M. Liles ${ }^{51}$, R. Lindner ${ }^{37}$, C. Linn ${ }^{11}$, B. $\mathrm{Liu}^{3}$, G. Liu ${ }^{37}$, S. Lohn ${ }^{37}$, I. Longstaff50 , J.H. Lopes ${ }^{2}$, E. Lopez Asamar ${ }^{35}$, N. Lopez-March ${ }^{38}$, H. Lu $^{3}$, D. Lucchesi ${ }^{21, q}$, J. Luisier ${ }^{38}$, H. Luo ${ }^{49}$, F. Machefert ${ }^{7}$, I.V. Machikhiliyan ${ }^{4,30}$, F. Maciuc ${ }^{28}$, O. $\mathrm{Maev}^{29,37}$, S. Malde ${ }^{54}$, G. Manca ${ }^{15, d}$, G. Mancinelli ${ }^{6}$, U. Marconi ${ }^{14}$, R. Märki ${ }^{38}$, J. Marks ${ }^{11}$, G. Martellotti ${ }^{24}$, A. Martens ${ }^{8}$, L. Martin ${ }^{54}$, A. Martín Sánchez ${ }^{7}$, M. Martinelli ${ }^{40}$, D. Martinez Santos ${ }^{41}$, D. Martins Tostes ${ }^{2}$, A. Massafferri ${ }^{1}$, R. Matev ${ }^{37}$, Z. Mathe ${ }^{37}$, C. Matteuzzi ${ }^{20}$, E. Maurice $^{6}$, A. Mazurov ${ }^{16,32,37, e}$, J. McCarthy ${ }^{44}$, A. McNab ${ }^{53}$, R. McNulty ${ }^{12}$, B. Meadows ${ }^{56,54}$, F. Meier ${ }^{9}$, M. Meissner ${ }^{11}$, M. Merk ${ }^{40}$, D.A. Milanes ${ }^{8}$, M.-N. Minard ${ }^{4}$, J. Molina Rodriguez ${ }^{58}$, S. Monteil ${ }^{5}$, D. Moran ${ }^{53}$, P. Morawski ${ }^{25}$, M.J. Morello ${ }^{22, s}$, R. Mountain ${ }^{57}$, I. Mous ${ }^{40}$, F. Muheim ${ }^{49}$,

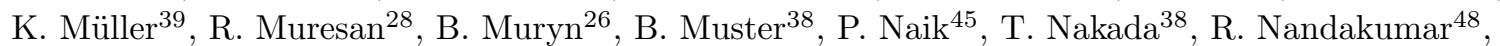
I. Nasteva ${ }^{1}$, M. Needham ${ }^{49}$, N. Neufeld ${ }^{37}$, A.D. Nguyen ${ }^{38}$, T.D. Nguyen ${ }^{38}$, C. Nguyen-Mau ${ }^{38, p}$, M. Nicol ${ }^{7}$, V. Niess ${ }^{5}$, R. Niet ${ }^{9}$, N. Nikitin ${ }^{31}$, T. Nikodem ${ }^{11}$, A. Nomerotski ${ }^{54}$, A. Novoselov ${ }^{34}$, A. Oblakowska-Mucha ${ }^{26}$, V. Obraztsov ${ }^{34}$, S. Oggero $^{40}, \mathrm{~S}$ Ogilvy $^{50}$, O. Okhrimenko ${ }^{43}$, R. Oldeman ${ }^{15, d}$, M. Orlandea ${ }^{28}$, J.M. Otalora Goicochea ${ }^{2}$, P. Owen ${ }^{52}$, A. Oyanguren ${ }^{35, o}$, B.K. Pal ${ }^{57}$, A. Palano ${ }^{13, b}$, M. Palutan ${ }^{18}$, J. Panman ${ }^{37}$, A. Papanestis ${ }^{48}$, M. Pappagallo ${ }^{50}$, C. $P_{a r k e s}^{53}$, C.J. Parkinson ${ }^{52}$, G. Passaleva ${ }^{17}$, G.D. Patel ${ }^{51}$, M. Patel ${ }^{52}$, G.N. Patrick ${ }^{48}$, C. Patrignani ${ }^{19, i}$, C. Pavel-Nicorescu ${ }^{28}$, A. Pazos Alvarez ${ }^{36}$, A. Pellegrino ${ }^{40}$, G. Penso ${ }^{24, l}$, M. Pepe Altarelli ${ }^{37}$, S. Perazzini ${ }^{14, c}$, D.L. Perego ${ }^{20, j}$, E. Perez Trigo ${ }^{36}$, A. Pérez-Calero Yzquierdo ${ }^{35}$,

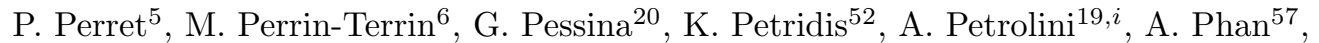

E. Picatoste Olloqui ${ }^{35}$, B. Pietrzyk ${ }^{4}$, T. Pilař ${ }^{47}$, D. Pinci ${ }^{24}$, S. Playfer ${ }^{49}$, M. Plo Casasus ${ }^{36}$, F. Polci ${ }^{8}$, G. Polok ${ }^{25}$, A. Poluektov ${ }^{47,33}$, E. Polycarpo ${ }^{2}$, D. Popov ${ }^{10}$, B. Popovici ${ }^{28}$, C. Potterat ${ }^{35}$, A. Powell ${ }^{54}$, J. Prisciandaro ${ }^{38}$, V. Pugatch ${ }^{43}$, A. Puig Navarro ${ }^{38}$, G. Punzi ${ }^{22, r}$, W. Qian ${ }^{4}$, J.H. Rademacker ${ }^{45}$, B. Rakotomiaramanana ${ }^{38}$, M.S. Rangel $^{2}$, I. Raniuk ${ }^{42}$, N. Rauschmayr ${ }^{37}$, G. Raven ${ }^{41}$, S. Redford ${ }^{54}$, M.M. Reid ${ }^{47}$, A.C. dos Reis ${ }^{1}$, S. Ricciardi ${ }^{48}$, A. Richards $^{52}$, K. Rinnert ${ }^{51}$, V. Rives Molina ${ }^{35}$, D.A. Roa Romero ${ }^{5}$, P. Robbe $^{7}$, E. Rodrigues ${ }^{53}$, P. Rodriguez Perez $^{36}$,

S. Roiser ${ }^{37}$, V. Romanovsky ${ }^{34}$, A. Romero Vidal ${ }^{36}$, J. Rouvinet ${ }^{38}$, T. Ruf ${ }^{37}$, F. Ruffini ${ }^{22}$, H. Ruiz ${ }^{35}$, P. Ruiz Valls ${ }^{35, o}$, G. Sabatino ${ }^{24, k}$, J.J. Saborido Silva ${ }^{36}$, N. Sagidova ${ }^{29}$, P. Sail ${ }^{50}$, B. Saitta ${ }^{15, d}$, C. Salzmann ${ }^{39}$, B. Sanmartin Sedes $^{36}$, M. Sannino ${ }^{19, i}$, R. Santacesaria ${ }^{24}$, C. Santamarina Rios ${ }^{36}$, E. Santovetti ${ }^{23, k}$, M. Sapunov ${ }^{6}$, A. Sarti ${ }^{18, l}$, C. Satriano ${ }^{24, m}$, A. Satta ${ }^{23}$, M. Savrie ${ }^{16, e}$, D. Savrina ${ }^{30,31}$, P. Schaack ${ }^{52}$, M. Schiller ${ }^{41}$, H. Schindler ${ }^{37}$, M. Schlupp ${ }^{9}$, M. Schmelling ${ }^{10}$, B. Schmidt ${ }^{37}$, O. Schneider ${ }^{38}$, A. Schopper ${ }^{37}$, M.-H. Schune ${ }^{7}$, R. Schwemmer ${ }^{37}$, B. Sciascia ${ }^{18}$, A. Sciubba ${ }^{24}$, M. Seco ${ }^{36}$, A. Semennikov ${ }^{30}$, K. Senderowska ${ }^{26}$, I. Sepp ${ }^{52}$, N. Serra ${ }^{39}$, J. Serrano ${ }^{6}$, P. Seyfert ${ }^{11}$, M. Shapkin ${ }^{34}$, I. Shapoval ${ }^{16,42}$, P. Shatalov ${ }^{30}$, Y. Shcheglov ${ }^{29}$, T. Shears ${ }^{51,37}$, L. Shekhtman ${ }^{33}$, O. Shevchenko ${ }^{42}$, V. Shevchenko ${ }^{30}$, A. Shires ${ }^{52}$, R. Silva Coutinho ${ }^{47}$, T. Skwarnicki ${ }^{57}$, N.A. Smith ${ }^{51}$, E. Smith ${ }^{54,48}$, M. Smith ${ }^{53}$, M.D. Sokoloff ${ }^{56}$, F.J.P. Soler ${ }^{50}$, F. Soomro ${ }^{18}$, D. Souza ${ }^{45}$, B. Souza De Paula ${ }^{2}$, B. Spaan ${ }^{9}$, A. Sparkes ${ }^{49}$, P. Spradlin ${ }^{50}$, F. Stagni ${ }^{37}$, S. Stahl ${ }^{11}$, O. Steinkamp ${ }^{39}$, S. Stoica ${ }^{28}$, S. Stone ${ }^{57}$, B. Storaci ${ }^{39}$, M. Straticiuc ${ }^{28}$, U. Straumann ${ }^{39}$, V.K. Subbiah ${ }^{37}$, S. Swientek ${ }^{9}$, V. Syropoulos ${ }^{41}$, M. Szczekowski ${ }^{27}$, P. Szczypka ${ }^{38,37}$, T. Szumlak ${ }^{26}$, S. T'Jampens ${ }^{4}$, M. Teklishyn ${ }^{7}$, E. Teodorescu ${ }^{28}$, F. Teubert ${ }^{37}$, C. Thomas ${ }^{54}$, E. Thomas $^{37}$, J. van Tilburg ${ }^{11}$, V. Tisserand ${ }^{4}$, M. Tobin ${ }^{38}$, S. Tolk ${ }^{41}$, D. Tonelli ${ }^{37}$, S. Topp-Joergensen ${ }^{54}$,

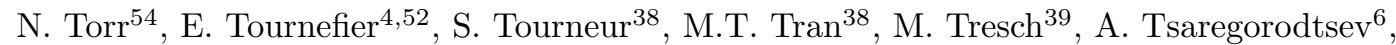
P. Tsopelas ${ }^{40}$, N. Tuning ${ }^{40}$, M. Ubeda Garcia ${ }^{37}$, A. Ukleja ${ }^{27}$, D. Unner ${ }^{53}$, U. Uwer ${ }^{11}$, V. Vagnoni ${ }^{14}$, G. Valenti ${ }^{14}$, R. Vazquez Gomez ${ }^{35}$, P. Vazquez Regueiro ${ }^{36}$, S. Vecchi ${ }^{16}$, J.J. Velthuis ${ }^{45}$, M. Veltri ${ }^{17, g}$, G. Veneziano ${ }^{38}$, M. Vesterinen ${ }^{37}$, B. Viaud ${ }^{7}$, D. Vieira ${ }^{2}$, X. Vilasis-Cardona ${ }^{35, n}$, 


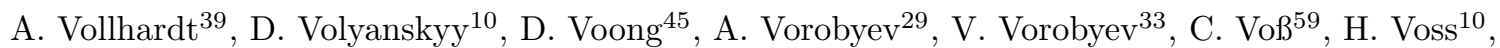
R. Waldi ${ }^{59}$, R. Wallace ${ }^{12}$, S. Wandernoth ${ }^{11}$, J. Wang ${ }^{57}$, D.R. Ward ${ }^{46}$, N.K. Watson ${ }^{44}$,

A.D. Webber ${ }^{53}$, D. Websdale ${ }^{52}$, M. Whitehead ${ }^{47}$, J. Wicht ${ }^{37}$, J. Wiechczynski ${ }^{25}$, D. Wiedner ${ }^{11}$, L. Wiggers ${ }^{40}$, G. Wilkinson ${ }^{54}$, M.P. Williams ${ }^{47,48}$, M. Williams ${ }^{55}$, F.F. Wilson ${ }^{48}$, J. Wishahi ${ }^{9}$, M. Witek ${ }^{25}$, S.A. Wotton ${ }^{46}$, S. Wright ${ }^{46}$, S. $\mathrm{Wu}^{3}$, K. Wyllie ${ }^{37}$, Y. Xie ${ }^{49,37}$, F. Xing ${ }^{54}$, Z. Xing ${ }^{57}$, Z. Yang ${ }^{3}$, R. Young ${ }^{49}$, X. Yuan ${ }^{3}$, O. Yushchenko ${ }^{34}$, M. Zangoli ${ }^{14}$, M. Zavertyaev ${ }^{10, a}$, F. Zhang ${ }^{3}$, L. Zhang ${ }^{57}$, W.C. Zhang ${ }^{12}$, Y. Zhang ${ }^{3}$, A. Zhelezov ${ }^{11}$, A. Zhokhov ${ }^{30}$, L. Zhong ${ }^{3}$, A. Zvyagin ${ }^{37}$.

1 Centro Brasileiro de Pesquisas Físicas (CBPF), Rio de Janeiro, Brazil

2 Universidade Federal do Rio de Janeiro (UFRJ), Rio de Janeiro, Brazil

3 Center for High Energy Physics, Tsinghua University, Beijing, China

4 LAPP, Université de Savoie, CNRS/IN2P3, Annecy-Le-Vieux, France

5 Clermont Université, Université Blaise Pascal, CNRS/IN2P3, LPC, Clermont-Ferrand, France

6 CPPM, Aix-Marseille Université, CNRS/IN2P3, Marseille, France

7 LAL, Université Paris-Sud, CNRS/IN2P3, Orsay, France

8 LPNHE, Université Pierre et Marie Curie, Université Paris Diderot, CNRS/IN2P3, Paris, France

9 Fakultät Physik, Technische Universität Dortmund, Dortmund, Germany

10 Max-Planck-Institut für Kernphysik (MPIK), Heidelberg, Germany

11 Physikalisches Institut, Ruprecht-Karls-Universität Heidelberg, Heidelberg, Germany

12 School of Physics, University College Dublin, Dublin, Ireland

13 Sezione INFN di Bari, Bari, Italy

14 Sezione INFN di Bologna, Bologna, Italy

15 Sezione INFN di Cagliari, Cagliari, Italy

16 Sezione INFN di Ferrara, Ferrara, Italy

17 Sezione INFN di Firenze, Firenze, Italy

18 Laboratori Nazionali dell'INFN di Frascati, Frascati, Italy

19 Sezione INFN di Genova, Genova, Italy

20 Sezione INFN di Milano Bicocca, Milano, Italy

21 Sezione INFN di Padova, Padova, Italy

22 Sezione INFN di Pisa, Pisa, Italy

23 Sezione INFN di Roma Tor Vergata, Roma, Italy

24 Sezione INFN di Roma La Sapienza, Roma, Italy

25 Henryk Niewodniczanski Institute of Nuclear Physics Polish Academy of Sciences, Kraków, Poland

${ }^{26}$ AGH - University of Science and Technology, Faculty of Physics and Applied Computer Science, Kraków, Poland

27 National Center for Nuclear Research (NCBJ), Warsaw, Poland

28 Horia Hulubei National Institute of Physics and Nuclear Engineering, Bucharest-Magurele, Romania

29 Petersburg Nuclear Physics Institute (PNPI), Gatchina, Russia

30 Institute of Theoretical and Experimental Physics (ITEP), Moscow, Russia

31 Institute of Nuclear Physics, Moscow State University (SINP MSU), Moscow, Russia

32 Institute for Nuclear Research of the Russian Academy of Sciences (INR RAN), Moscow, Russia

33 Budker Institute of Nuclear Physics (SB RAS) and Novosibirsk State University, Novosibirsk, Russia

34 Institute for High Energy Physics (IHEP), Protvino, Russia

35 Universitat de Barcelona, Barcelona, Spain

36 Universidad de Santiago de Compostela, Santiago de Compostela, Spain

37 European Organization for Nuclear Research (CERN), Geneva, Switzerland

38 Ecole Polytechnique Fédérale de Lausanne (EPFL), Lausanne, Switzerland

39 Physik-Institut, Universität Zürich, Zürich, Switzerland

40 Nikhef National Institute for Subatomic Physics, Amsterdam, The Netherlands

41 Nikhef National Institute for Subatomic Physics and VU University Amsterdam, Amsterdam, The Netherlands

42 NSC Kharkiv Institute of Physics and Technology (NSC KIPT), Kharkiv, Ukraine 
Institute for Nuclear Research of the National Academy of Sciences (KINR), Kyiv, Ukraine

44 University of Birmingham, Birmingham, United Kingdom

45 H.H. Wills Physics Laboratory, University of Bristol, Bristol, United Kingdom

${ }^{46}$ Cavendish Laboratory, University of Cambridge, Cambridge, United Kingdom

47 Department of Physics, University of Warwick, Coventry, United Kingdom

48 STFC Rutherford Appleton Laboratory, Didcot, United Kingdom

49 School of Physics and Astronomy, University of Edinburgh, Edinburgh, United Kingdom

50 School of Physics and Astronomy, University of Glasgow, Glasgow, United Kingdom

51 Oliver Lodge Laboratory, University of Liverpool, Liverpool, United Kingdom

52 Imperial College London, London, United Kingdom

53 School of Physics and Astronomy, University of Manchester, Manchester, United Kingdom

54 Department of Physics, University of Oxford, Oxford, United Kingdom

55 Massachusetts Institute of Technology, Cambridge, MA, United States

56 University of Cincinnati, Cincinnati, OH, United States

57 Syracuse University, Syracuse, NY, United States

58 Pontifícia Universidade Católica do Rio de Janeiro (PUC-Rio), Rio de Janeiro, Brazil, associated to ${ }^{2}$

59 Institut für Physik, Universität Rostock, Rostock, Germany, associated to ${ }^{11}$

${ }^{a}$ P.N. Lebedev Physical Institute, Russian Academy of Science (LPI RAS), Moscow, Russia

${ }^{b}$ Università di Bari, Bari, Italy

c Università di Bologna, Bologna, Italy

d Università di Cagliari, Cagliari, Italy

e Università di Ferrara, Ferrara, Italy

${ }^{f}$ Università di Firenze, Firenze, Italy

$g$ Università di Urbino, Urbino, Italy

${ }^{h}$ Università di Modena e Reggio Emilia, Modena, Italy

${ }^{i}$ Università di Genova, Genova, Italy

${ }^{j}$ Università di Milano Bicocca, Milano, Italy

${ }^{k}$ Università di Roma Tor Vergata, Roma, Italy

$l$ Università di Roma La Sapienza, Roma, Italy

$m$ Università della Basilicata, Potenza, Italy

${ }^{n}$ LIFAELS, La Salle, Universitat Ramon Llull, Barcelona, Spain

- IFIC, Universitat de Valencia-CSIC, Valencia, Spain

${ }^{p}$ Hanoi University of Science, Hanoi, Viet Nam

${ }^{q}$ Università di Padova, Padova, Italy

$r$ Università di Pisa, Pisa, Italy

$s$ Scuola Normale Superiore, Pisa, Italy 